UVX 2008 (2009) 51-56

(C) EDP Sciences, 2009

DOI: $10.1051 / \mathrm{uvx} / 2009009$

\title{
Caractérisation spatio-temporelle d'un laser XUV injecté
}

\author{
J.-P. Goddet ${ }^{1}$, S. Sebban 1 , O. Guilbaud ${ }^{2}$, G. Maynard ${ }^{3}$, B. Cros $^{3}$, J. Gautier ${ }^{1}$, \\ Ph. Zeitoun ${ }^{1}$, C. Valentin ${ }^{1}$, F. Tissandier ${ }^{1}$, T. Marchenko ${ }^{1}$, G. Lambert ${ }^{1}$, \\ D. Benredjem², A. Boudaa ${ }^{3}$, A. Klisnick², D. Ros², S. Kazamias², \\ K. Cassou ${ }^{2,3}$, J. Habib², G. Jamelot ${ }^{2}$, J.-C. Lagron², D. Joyeux ${ }^{4}$, \\ S. De Rossi ${ }^{4}$, D. Phalippou ${ }^{4}$, F. Delmotte ${ }^{4}$, M.F. Ravet ${ }^{4}$, A. Calisti ${ }^{5}$, T. Mocek ${ }^{6}$, \\ M. Kozlová ${ }^{6}$ et K. Jakubczak ${ }^{6}$ \\ ${ }^{1}$ Laboratoire d'Optique Appliquée, Palaiseau, France \\ ${ }^{2}$ Laboratoire d'Interaction du Rayonnement $X$ avec la Matière, Orsay, France \\ ${ }^{3}$ Laboratoire des Gaz et des Plasmas, Orsay, France \\ ${ }^{4}$ Laboratoire Charles Fabry de I'Institut d'Optique, Orsay, France \\ ${ }^{5}$ Physique des Interactions loniques et Moléculaires, Marseille, France \\ ${ }^{6}$ Institute of Physics, Prague, Czech Republic
}

\begin{abstract}
Résumé. Depuis la démonstration expérimentale au LOA en 2004, d'un laser XUV (OFI) injecté par un faisceau d'harmoniques d'ordres élevés, de nouveaux résultats sur la caractérisation de cette source XUV ont récemment été obtenus. En effet, grâce au filtrage spatial des harmoniques par le milieu amplificateur, l'harmonique amplifié à $32.8 \mathrm{~nm}$ nous montre un profil spatiale gaussien avec une divergence de $0.67 \mathrm{mrad}$. La variation du front d'onde a été mesurée avec un senseur de front d'onde de type Hartmann et donne une valeur de $\lambda / 17 \mathrm{rms}$. Ce qui démontre que ce laser à $32.8 \mathrm{~nm}$ est limitée par la diffraction. La mesure de la cohérence temporelle donne un $\delta \lambda / \Lambda$ égal à $10^{-5}$ correspondant à une durée d'impulsion de l'ordre de $5 \mathrm{ps}$
\end{abstract}

\section{INTRODUCTION}

Le laser XUV (OFI) par injection d'harmoniques d'ordre élevé est une analogie directe du concept "oscillateur amplificateur" couramment utilisé pour les systèmes laser infrarouge de puissance. Ici, un faisceau d'harmoniques du faisceau IR joue le rôle d'un oscillateur qui est injecté et amplifié dans une colonne de plasma de krypton $8+$ en inversion de population. L'inversion de population se produit entre les transitions $3 \mathrm{~d} 94 \mathrm{p}(1 \mathrm{P} 1) \rightarrow 3 \mathrm{~d} 94 \mathrm{~d}(1 \mathrm{~S} 0)$ à $32.8 \mathrm{~nm}$. Ce régime offre des perspectives pour la réalisation d'une chaîne compacte dans le domaine XUV combinant à la fois l'énergie extraite de l'amplificateur ainsi que la haute qualité optique du faisceau harmonique. Nous présenterons ici les derniers résultats réalisés au Laboratoire d'Optique Appliquée sur la caractérisation spatio-temporelle de cette source laser XUV. Notamment sur la distribution d'énergie, la mesure du front d'onde ainsi que sur la mesure de la cohérence spatiale et temporelle serons présentées. De plus, nous avons réalisé des simulations qui montrent que la bonne qualité spatiale est due au filtrage spatial des harmoniques par le plasma amplificateur.

\section{MONTAGE EXPÉRIMENTAL}

Les expériences décrites ont été réalisées au Laboratoire d'Optique Appliquée à l'aide d'une chaîne laser multi-terawatt fonctionnant à $10 \mathrm{~Hz}$. Cette chaîne laser délivre deux faisceaux indépendants d'une durée d'impulsions de 35 fs à une longueur d'onde de $815 \mathrm{~nm}$. Le dispositif expérimental est représenté sur la Figure 1. Le premier faisceau fournit $500 \mathrm{~mJ}$ sur cible et est utilisé pour créer le milieu amplificateur. Le faisceau est focalisé par un miroir sphérique de 1 mètre de focale dans une cellule de 7,5 mm de long rempli avec 30 mbar de Krypton [2]. Le deuxième faisceau laser d'environ $10 \mathrm{~mJ}$ est utilisé pour générer 


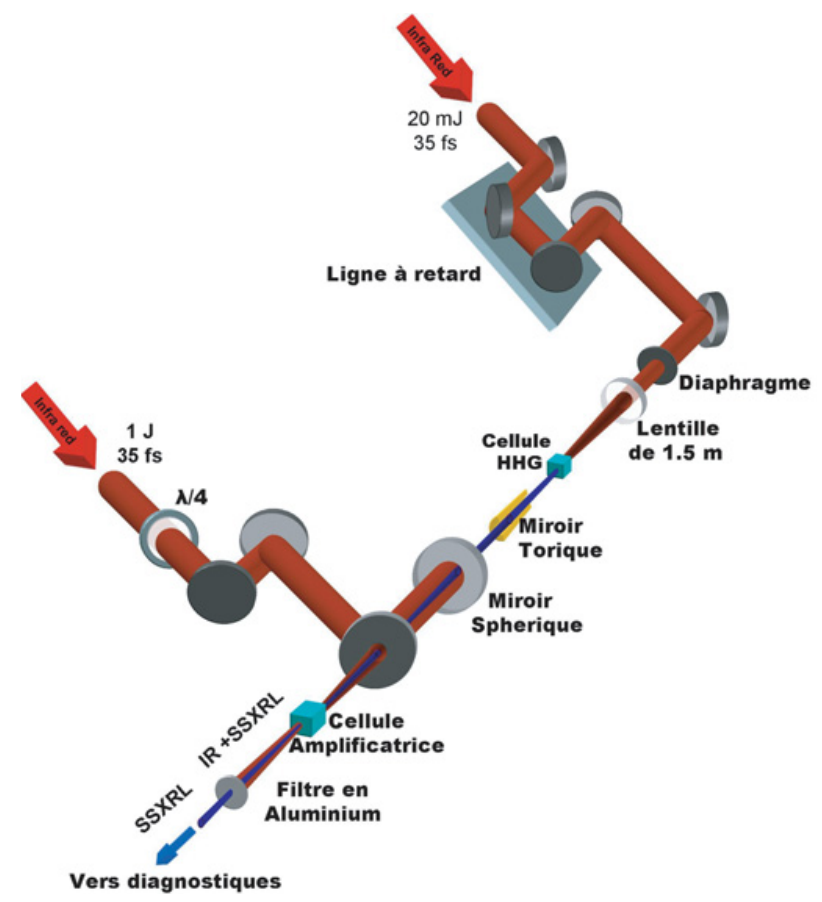

Figure 1. Montage expérimental du laser XUV injecté.

le faisceau harmonique. Les harmoniques sont générées dans une autre cellule de $7 \mathrm{~mm}$ rempli de 30 mbars d'argon, au moyen d'une lentille sphérique qui possède une focale de 1,5 m. Un miroir torique en incidence rasant est utilisé pour imager la sortie de la source harmonique avec un grossissement de 1,5 à l'entrée de l'amplificateur. Une ligne à retard est utilisée sur la ligne harmonique pour synchroniser les deux faisceaux. Le laser XUV injecté est ensuite envoyé vers différents diagnostiques qui seront décrit par la suite.

\section{PROFIL SPATIAL}

La mesure de la distribution d'énergie a été mesuré à l'aide d'une caméra CCD XUV situé à $2.55 \mathrm{~m}$ de la cellule amplificatrice. La Figure 2(a) nous montre le profil d'intensité des harmoniques. A cause d'un léger desalignement du miroir torique (optique de couplage entre les harmoniques et l'amplificateur), les harmoniques ont un profil astigmatique avec une divergence horizontal et vertical respectivement de $1.32 \mathrm{mrad}$ et $0.48 \mathrm{mrad}$. Comme le montre la Figure 2(b) le profil d'intensité dule laser XUV injecté est remarquablement différent. En effet, le profil de l'harmonique amplifié, en plus d'être très intense et monochromatique, est très proche d'un forme gaussienne et possède une divergence très faible de $0.67 \mathrm{mrad}\left(\right.$ demi angle à $\left.1 / \mathrm{e}^{2}\right)$. Pour mieux comprendre cette amélioration spatiale, nous avons réalisé des mesures de front d'onde.

\section{MESURE DE FRONT D'ONDE}

\subsection{Résultats}

La mesure du front d'onde a été réalisée à l'aide d'un senseur de front de type Hartmann. A l'aide d'une matrice de trous, nous échantillonnons le faisceau incident en une multitude de sous faisceaux. De ce fait, 

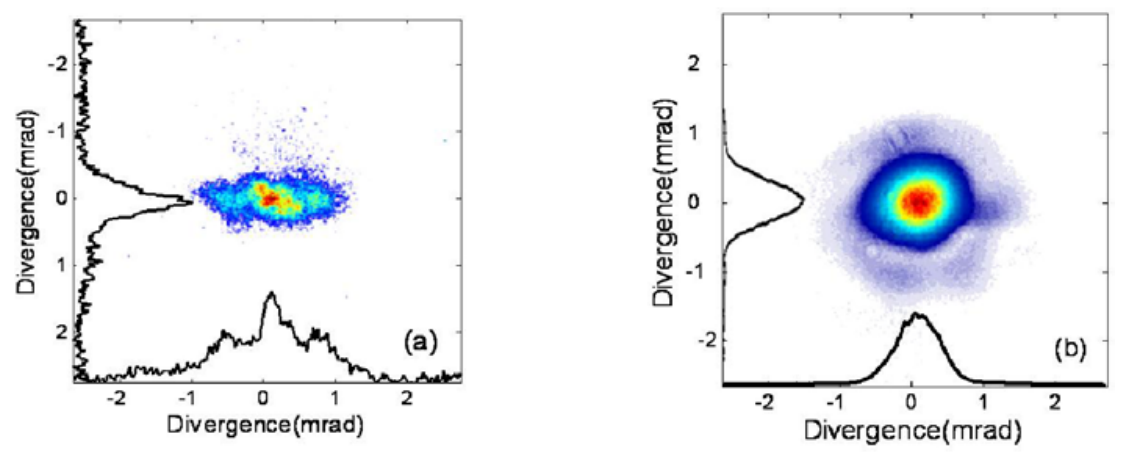

Figure 2. Profil spatial pour de l'injecteur harmonique (a) et pour le laser XUV injecté (b).
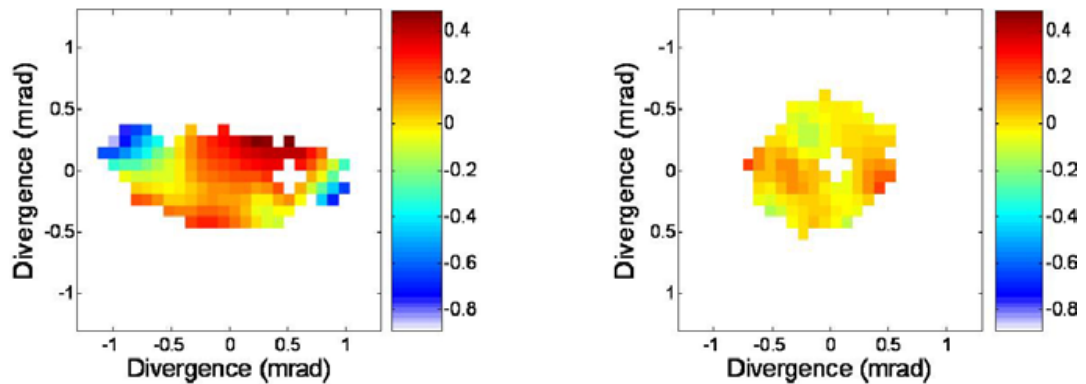

Figure 3. Mesure de la variation du front d'onde des harmoniques (a) et des harmoniques amplifiés (b).

chaque sous faisceaux se propage librement suivant une direction qui dépend de la courbure locale du front d'onde au niveau du trou. Ces sous faisceaux arrivent sur un détecteur, dans notre cas il s'agit d'une caméra CCD, pour former dans ce plan de détection un réseau de taches. La distance entre la plaque de trou et le détecteur étant connu, une mesure de l'écart de ces taches dans le plan du détecteur par rapport à des positions dites de références permet alors d'obtenir une cartographie bidimensionnelle des pentes locales du front d'onde, c'est-à-dire les dérivées locales de la surface d'onde. La Figure 3(a) nous donne un exemple typique des valeurs mesurées par le senseur de front d'onde pour les harmoniques. Les harmoniques possèdent de grandes variations dans la mesure de la phase avec une variation moyenne de $0.3 \lambda(\mathrm{RMS})$. La Figure 3(b) nous montre les variations du front d'onde dans le cas du laser XUV injecté. Il y a un vrai contraste entre les valeurs des harmoniques avant et après amplification. En effet, les variations du front d'onde pour le laser XUV injecté sont plus faible avec une valeur Rms de $0.058 \lambda$ ce qui correspond à $\lambda / 17(1.9 \mathrm{~nm})$. D'après le critère de Maréchal [2], le faisceau amplifié est ici limité par la diffraction.

\subsection{Reconstruction de la source}

Avec le senseur de front d'onde, nous avons simultanément mesuré l'intensité et la phase du faisceau XUV, ce qui nous a permis de calculer la valeur de l'amplitude complexe au niveau du détecteur. Ensuite, nous pouvons reconstruire l'amplitude complexe des différentes sources où on le souhaite sur l'axe du laser. Les résultats de ce calcul en termes d'intensité sont reportés sur la Figure 4(a) pour les harmoniques à l'entrée de la colonne de plasma amplificatrice. Nous pouvons observé un profil ovale avec des dimensions de $55 \mu \mathrm{m} \times 116 \mu \mathrm{m}$ (à 1/e2). En utilisant la même méthode, la Figure 4(b) nous montre le profil d'intensité de l'harmonique amplifié à la sortie du plasma. Le profil est beaucoup plus 
symétrique et les dimensions sont de $58 \mu \mathrm{m} \times 77 \mu \mathrm{m}$. Les dimensions du laser XUV injecté à la sortie du plasma sont plus petites que les harmoniques avant amplification. Ce qui montre que le plasma amplificateur a joué le rôle de filtre spatial.
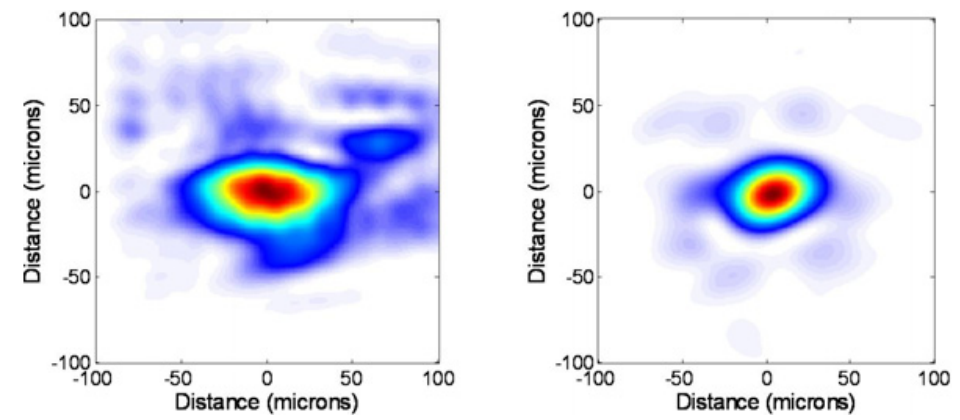

Figure 4. Reconstruction à l'entrée du plasma amplificateur du profil d'intensité des harmoniques (a) et celle des harmoniques amplifiés (b).

\subsection{Modèle numérique simple pour expliquer le filtrage spatial par le gain}

Pour comprendre cet effet de filtrage, nous avons effectué une analyse théorique simple du couplage spatiale des harmoniques dans le plasma amplificateur. L'amplitude complexe du laser XUV injecté, à la sortie du plasma prend la forme simple suivante :

$$
E_{s s x r l}(x, y, z=L)=\sqrt{G} E_{H}(x, y, z=0) H\left(R-\sqrt{x^{2}+y^{2}}\right)
$$

Avec $\mathrm{G}$ le facteur d'amplification, $\mathrm{H}(\mathrm{u})$ la fonction d'Heaviside et $E_{H}$ l'amplitude complexe pour les harmoniques d'ordres élevés. Nous voyons dans l'équation que l'influence du plasma amplificateur sur les harmoniques est identique à celui d'un diaphragme circulaire. Après l'avoir obtenu, nous avons calculé l'amplitude complexe au niveau du détecteur de front d'onde. Ces calcules ont été répétés pour différents rayons de plasma allant de $15 \mu \mathrm{m}$ à $1000 \mu \mathrm{m}$.
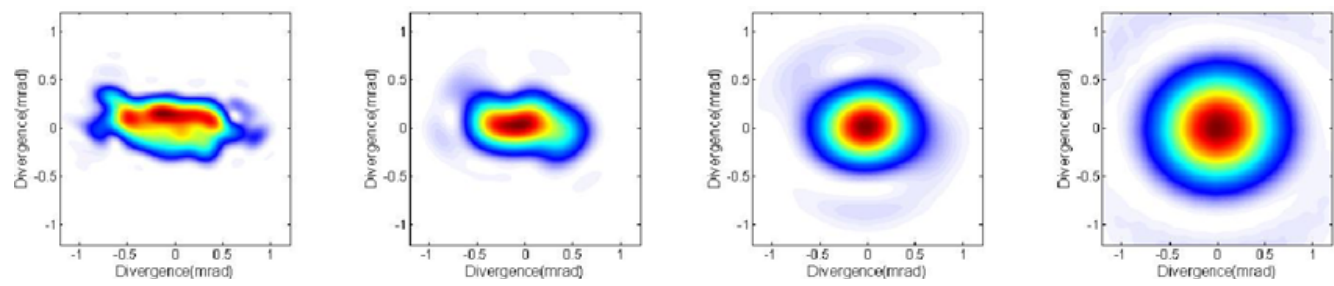

Figure 5. Reconstruction 2D du champ lointain des harmoniques après le filtrage spatial pour différentes tailles de plasmas : (a) $200 \mu \mathrm{m}$, (b) $100 \mu \mathrm{m}$, (c) $60 \mu \mathrm{m}$, (d) $40 \mu \mathrm{m}$.

La Figure 5 nous montre la distribution de l'intensité déterminée par $E_{s s x r l}\left(x, y, z=z_{H}\right)$ en fonction de différents diamètres du plasma. En réduisant le diamètre, le faisceau évolue d'une forme astigmatique à un profil parfaitement circulaire. Pour un plasma de diamètre de $60 \mu \mathrm{m}$ (Figure 2(c)), la divergence du laser XUV injecté simulée est de $0.46 \mathrm{mrad} \times 0.57 \mathrm{mrad}$, ce qui est en bon accord avec nos résultats expérimentaux mesurés à $0.67 \mathrm{mrad}$ (Figure 2(b)). Pour des diamètres plus petits que $50 \mu \mathrm{m}$ comme sur la Figure 2(d), la divergence dule laser XUV injecté augmente à cause des l'effets de diffraction.

Les variations du front d'onde en fonction du diamètre du plasma sont reportées dans la Figure 6. La limite de diffraction $(\lambda / 14)$ est atteinte pour un diamètre de $74 \mu \mathrm{m}$. En accord avec nos mesures 
expérimentales du front d'onde à $\lambda / 17$, les harmoniques sont filtrés par une colonne de plasma de l'ordre de $60 \mu \mathrm{m}$ de diamètre. Cette valeur est en bon accord avec les résultats obtenus par la divergence et la distribution d'énergie du faisceau.

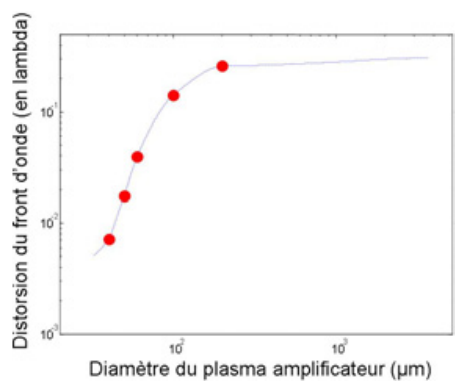

Figure 6. Variation du front d'onde calculée pour le laser XUV injecté en fonction du diamètre du plasma amplificateur.

\section{COHÉRENCE SPATIALE}

Ensuite, nous avons mesuré la cohérence spatiale du laser XUV amplifié grâce à un système de fentes d'Young comportant 4 espacements de fentes allant de $115 \mu \mathrm{m}$ à $423 \mu \mathrm{m}$. Nous avons aussi mesuré la cohérence du rayonnement à $32.8 \mathrm{~nm}$ émis par le plasma et du faisceau harmonique non amplifié pour les comparer avec le faisceau du laser XUV injecté. Les valeurs de la visibilité en fonction longueur de séparation des fentes pour le laser XUV injecté, le faisceau ASE, et les harmoniques seules sont reportées dans la Figure 7.

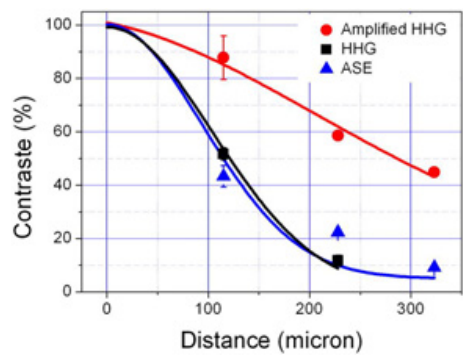

Figure 7. Mesure du contraste pour différentes longueurs d'espacement entre les fentes pour les harmoniques avant amplification (en rouge), l'ASE (en bleu) et pour le laser XUV injecté (en noir).

La mesure de la visibilité montre qu'une amélioration spectaculaire de la cohérence transverse du rayonnement à $32,8 \mathrm{~nm}$ a été obtenue après amplification. Pour le faisceau amplifié, la longueur de cohérence atteint $232 \mu \mathrm{m}$, ce qui correspond à $55 \%$ du disque central calculé à mi-hauteur du faisceau. Ici, nous définissons la longueur de cohérence spatiale Rc comme la longueur pour lequel la visibilité est diminuée d'un facteur 1/e. En comparaison, le faisceau d'harmoniques possède une longueur de cohérent égal à $102 \mu \mathrm{m}$, ce qui correspond à environ un cinquième du diamètre du faisceau calculé à mi-hauteur au niveau des fentes. La longueur de cohérente de l'émission l'ASE est d'environ $98 \mu \mathrm{m}$, ce qui est environ 122 fois plus petit que le diamètre du faisceau mesuré au niveau de fentes. Le faisceau amplifié possède donc une excellente cohérence spatiale. Ce résultat est la conséquence directe du filtrage des harmoniques par le plasma amplificateur [3].

\section{COHÉRENCE TEMPORELLE}

La cohérence temporelle a été mesurée avec un interféromètre à bi-dièdre. La Figure 8 nous montre la mesure de visibilité des franges en fonction de la différence de marche entre les deux bras de l'interféromètre. Les données ont été ajustées par une fonction ce qui permet de déterminer un temps de 
cohérence de $5.4 \pm 0.3 \mathrm{ps}$, défini comme la différence de marche pour laquelle la visibilité maximum est atténuée d'un facteur 1/e. L'évolution du profil correspond à un profil spectral gaussien avec une largeur spectrale égal à $\Delta \lambda=3.4 \pm 0.2 \mathrm{~mA}$ ou encore un $\Delta v=9.6 \pm 0.210^{10} \mathrm{~Hz}$.

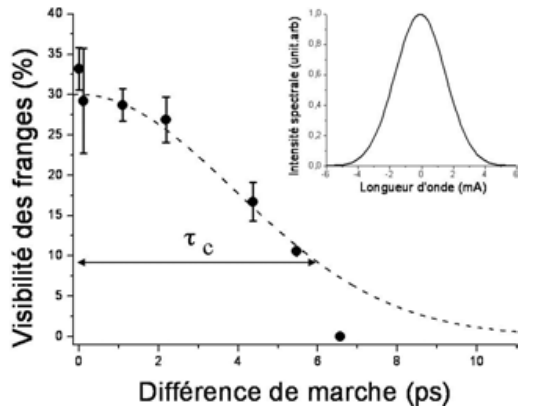

Figure 8. Visibilité des franges d'interférences en fonction du délai entre les deux bras de l'interféromètre. La courbe en pointillé représente le tracé d'une gaussienne qui suit les points expérimentaux. En haut à droite nous avons le spectre par transformée de Fourier de cette courbe gaussienne.

La dynamique temporelle du gain du plasma amplificateur a été mesurée en faisant varier le délai entre le moment où l'on a créé le plasma amplificateur et le moment où l'on injecte le faisceau harmonique à amplifier. La durée totale du gain étant de l'ordre de $8 \mathrm{ps}$ et le délai de l'injection de 3 ps, cela signifie que la durée d'impulsion maximale des harmoniques amplifiés sera limitée par la durée du gain après l'injection des harmoniques, c'est à dire $5 \mathrm{ps}$. Le fait que le temps de cohérence soit à peu près égal à la durée maximum de l'impulsion amplifiée ( durée du gain) montre que cette source est en limite de Fourier.

\section{CONCLUSION}

En amplifiant un faisceau harmonique dans un plasma OFI en inversion de population, nous avons réalisé une source laser XUV à $32.8 \mathrm{~nm}$ limité par la diffraction et en limite de Fourier. Cette source laser très cohérente dans le domaine XUV peut être un très bon outil pour de nombreuses applications comme la lithographie, l'interférométrie et l'holographie.

\section{Références}

[1] Ph. Zeitoun et al., Nature 431, (2004) 466.

[2] A. Marechal, Rev. D'Optique 26, (1947) 257.

[3] J. Ph. Goddet et al., Opt. Lett. 32, (2007) 1498. 